\title{
Integrating advance care planning as part of comprehensive geriatric assessment for hospitalised frail elderly patients: findings of a cross-sectional study
}

\author{
King Fan $\underline{\text { Yip }}^{1}$, MBBS, MRCP, Ting Hway Wong ${ }^{2,3}$, MBBCh, FRCSEd, Sharifah Munirah Alhamid ${ }^{4}$, MBBS, MRCP, \\ Nivedita Nadkarni ${ }^{5}$, PhD, Charlene Kay Gek $\underline{\text { Tann }}^{6}$, MB BCh BAO, Amanda Pang ${ }^{7}$, BSc, Chuen Chai Dennis Seow ${ }^{1}$, MBBS, FRCP
}

\begin{abstract}
INTRODUCTION The integration of advance care planning (ACP) as part of the comprehensive geriatric assessment (CGA) of hospitalised frail elderly patients, together with the clinical and demographic factors that determine successful ACP discussion, has not been previously explored.

METHODS A cross-sectional study on patients and family caregivers admitted under the geriatric medicine department of a tertiary hospital was conducted from October 2015 to December 2016.

RESULTS Among 311 eligible patients, 116 (37.3\%) patients completed ACP discussion while 166 (53.4\%) patients declined, with 62 (37.3\%) of the decliners providing reasons for refusal. Univariate logistic regression analysis showed that older age, higher Charlson Comorbidity Index, poorer functional status and cognitive impairment had statistically significant associations with agreeing to ACP discussion $(p<0.05)$. On multivariate logistic regression analysis, only poorer functional status was significantly associated (odds ratio 2.22 [95\% confidence interval $1.27-3.87$ ]; $p=0.005$ ). Among those who completed ACP discussion, a majority declined cardiopulmonary resuscitation (79.3\%), preferred limited medical intervention or comfort care (82.8\%), and opted for blood transfusion (62.9\%), antibiotics (73.3\%) and intravenous fluid (74.1\%) but declined haemodialysis (50.9\%). Decision-making was divided for enteral feeding. Among decliners, the main reasons for refusal were 'not keen' (33.9\%), 'deferring to doctors' decision' (11.3\%) and 'lack of ACP awareness' (11.3\%).

CONCLUSION The feasibility and utility of integrating ACP as part of CGA has been demonstrated. Poorer functional status is significantly associated with successful ACP discussion. Greater public education on end-of-life care choices (besides cardiopulmonary resuscitation) and follow-up with decliners are recommended.
\end{abstract}

Keywords: advance care planning, comprehensive geriatric assessment, elderly, end-of-life care choices, hospitalised

\section{INTRODUCTION}

Advance care planning (ACP) is a vital process that determines the type and quality of care that one receives towards the end of life (EOL). It involves voluntary discussions that facilitate understanding of patients' health status, values, beliefs and preferences in order to make appropriate healthcare decisions that are aligned with their wishes. The main stakeholders in an ACP conversation include patients, family caregivers (FCGs) and healthcare professionals. Successful completion of ACP has been associated with older age, higher education, higher income, and people with chronic disease and a regular source of care. ${ }^{(1)}$ In terms of outcomes, ACP has been shown to improve EOL care, patient and family satisfaction, and reduce stress, anxiety and depression in surviving relatives. ${ }^{(2)}$

In ageing Singapore, ACP discussion has heightened importance, as elderly patients have a higher incidence of multiple comorbidities, cognitive impairment, hospitalisation and death compared with other age groups. Local studies on ACP have revealed two areas of importance: first, the receptiveness and barriers to discussing ACP among patients and FCGs; and second, the types of EOL care choices made.

Regarding receptiveness and barriers, a local community survey revealed that only $37 \%$ of those aged 60 years and above had discussed death or dying with their loved ones. ${ }^{(3)}$ However, $90 \%$ in the same survey group were receptive towards a national conversation on death and dying. Barriers included awaiting others to initiate the dialogue, lack of knowledge to broach the topic and concerns about its impact on family members. An exploratory, descriptive study of FCGs of patients with advanced illness demonstrated low ACP awareness even among younger FCGs (mean age 43.2 years). ${ }^{(4)}$ In this earlier study, a frequently cited barrier to ACP discussion was the perception by FCGs that it was a sensitive and emotionally charged topic.

In terms of EOL care choices, there has been a diversity of findings across different settings, with a trend towards more conservative care choices among the institutionalised. In a study of elderly Chinese people at a day care centre, $67.4 \%$ of the participants preferred cardiopulmonary resuscitation (CPR), $62.8 \%$ wanted artificial ventilation, $55.8 \%$ opted for nasogastric feeding, $65.1 \%$ wanted intravenous hydration and $41.9 \%$ wanted renal dialysis for EOL care. ${ }^{(5)}$ A cross-sectional study comparing the attitudes towards EOL care among patients, relatives and healthcare professionals at a geriatric outpatient clinic found that $34 \%$ of patients opted for CPR compared to $50 \%$ of relatives, and $20 \%$ of patients wanted intubation and $26 \%$ chose nasogastric

${ }^{1}$ Department of Geriatric Medicine, ${ }^{2}$ Department of General Surgery, Singapore General Hospital, ${ }^{3}$ Duke-NUS Medical School, ${ }^{4}$ Department of Geriatric Medicine, Changi General Hospital, ${ }^{5}$ Centre for Quantitative Medicine, Duke-NUS Medical School, ${ }^{6}$ Ministry of Health Holdings, 'Department of Reproductive Medicine, KK Women's and Children's Hospital, Singapore

Correspondence: Dr King Fan Yip, Consultant, Department of Geriatric Medicine, Singapore General Hospital, Outram Road, Singapore 169608. yip.king.fan@singhealth.com.sg 
feeding. ${ }^{(6)}$ In a more recent study conducted at seven nursing homes, a majority preferred limited additional intervention at the nursing home, with escalation to the hospital if necessary (52.3\%), followed by limited additional intervention at the nursing home only $(36.5 \%){ }^{.7)}$ In this study, $93 \%$ of residents and their families declined $C P R$, while the most preferred place of death was the nursing home $(77 \%)$. A review of the local literature revealed that there has hitherto been no local study on ACP within the acutely hospitalised cohort.

Comprehensive geriatric assessment (CGA) is the cornerstone of good geriatric care. It is defined as a "multidimensional interdisciplinary diagnostic process focused on determining a frail elderly person's medical, psychological and functional capability in order to develop a coordinated and integrated plan for treatment and long-term follow-up ${ }^{\prime \prime}{ }^{(8)}$ The goals of CGA are to: generate interdisciplinary care plans; address physical, psychological and socioeconomic issues; improve survival rate; and establish better quality of life. However, the concept of integrating ACP as part of CGA for frail elderly people has not been previously explored.

We therefore set out to explore the outcomes of ACP advocacy and discussion among frail geriatric patients and their FCGs as part of CGA within the acute inpatient setting. The aims of the study were to examine: (a) the utility and outcomes of ACP discussion as part of CGA, together with the clinical and demographic factors associated with successful completion of such discussions; and (b) reasons for declining the discussion in the inpatient setting. We hypothesised that certain factors predisposed patients to being more open to ACP discussion when offered as part of a CGA.

\section{METHODS}

This was a cross-sectional study conducted on all patients admitted to the Department of Geriatric Medicine at Singapore General Hospital, a 1,700-bed tertiary teaching hospital, from 1 October 2015 to 31 December 2016.

Since November 2014, ACP has been integrated into existing CGA for all patients admitted at the department. This is a novel addition to traditional CGA, which encompasses the aspects of physical health, mental function, functional capacity, and social, environmental and economical resources. As geriatricians often establish a good rapport with their patients and FCGs, it was decided that such integration would reinforce the concept of EOL care as part of holistic patient management. Data collected in the CGA included demographics, geriatric syndromes, medical issues, functional status (activities of daily living [ADL] and instrumental ADL), cognitive assessment, social history and caregiving arrangement. The ACP components that were integrated included resuscitation status, usage of antibiotics and intravenous fluid, blood transfusion, haemodialysis, mode of feeding, and preferred place of medical care and death towards EOL. Reasons for declining ACP were documented as well.

All new team doctors rotated into the geriatric medicine department were educated on how to initiate and conduct ACP discussions with patients and/or their FCGs. This education took place on an almost monthly basis. ACP discussion only took place when patients were medically stable and lucid. This usually occurred on Day 3 of admission (with some variation) and was based on our clinical experience and observations that most patients required about three days for their medical condition(s) to stabilise. This was also congruent with a retrospective cohort study in the United States, which showed that the median time from admission to life-sustaining treatment limitation was 2.3 days for a subgroup of patients aged 65-84 years. ${ }^{(9)}$

Eligible patients were those aged 65 years and above admitted at the geriatric medicine department between 1 October 2015 and 31 December 2016. These included patients with geriatric syndromes who were transferred to the department from other specialties after inpatient geriatric medicine consultation. For cognitively impaired patients who lacked decision-making capacity, the ACP discussion was conducted with FCGs when present. Exclusion criteria included patients who were dangerously ill with extremely poor prognosis and close to demise, and cognitively impaired patients who were unable to make decisions and had no next-of-kin/surrogate decision maker or whose next-of-kin/surrogate decision maker was uncontactable/estranged.

Barthel Index ADL scores were used to assess patient premorbid functional status and were divided into four ADL groups according to International Statistical Classification of Diseases and Related Health Problems 10th revision diagnoses. ${ }^{(10)}$ Dementia staging was based on the Functional Assessment Staging Tool. ${ }^{(11)}$ Ethics approval for the study was obtained from the centralised institutional review board (reference no. 2016/2398).

\section{RESULTS}

Among the 322 patients under the care of the geriatric medicine department during the study period, 11 patients were excluded (Fig. 1). Of the remaining 311 patients, 145 (46.6\%) patients agreed to an ACP discussion and 166 (53.4\%) patients refused. The mean age was 81.2 years and $72.7 \%$ of patients were women. However, 29 patients who initially agreed to ACP did not proceed with the discussion; the remaining 116 patients completed it. Characteristics of patients who completed or declined the ACP discussion are presented in Table I.

On univariate logistic regression analysis, older age (odds ratio [OR] 1.04, 95\% confidence interval [CI] 1.01-1.07; $\mathrm{p}=0.015)$, higher Charlson Comorbidity Index (OR 1.15, 95\% Cl 1.02-1.31; $p=0.024$ ), poorer functional status (i.e. Barthel Index for $\mathrm{ADL}<80$; OR 3.05, 95\% Cl 1.86-4.99; $\mathrm{p}<0.001$ ) and cognitive impairment (OR 2.08, 95\% Cl 1.28-3.38; $p=0.003$ ) were significantly associated with patients or FCGs agreeing to ACP discussion. However, on multivariate logistic regression analysis, only poorer functional status (OR 2.22, 95\% Cl 1.27-3.87; $\mathrm{p}=0.005)$ was significantly associated with agreement to ACP discussion (Table II).

The reasons for declining ACP discussion are listed in Table III. A majority (33.9\%) were not keen but unable to elaborate. 'Deferring to doctor's decision' (11.3\%) and 'lack of ACP awareness/understanding' (11.3\%) were tied as the secondmost common reason. 


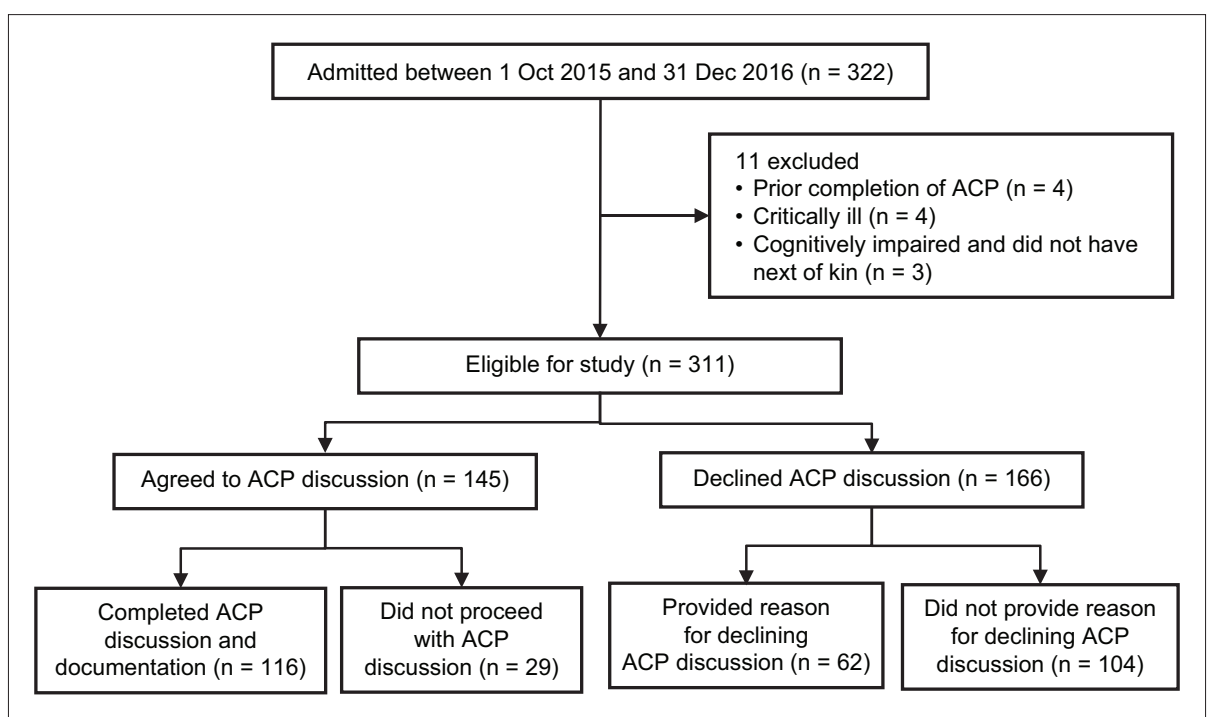

Fig. 1 Flowchart shows the consort diagram for the study. ACP: advance care planning

Table I. Characteristics of patients who completed or refused ACP discussion.

\begin{tabular}{|c|c|c|}
\hline \multirow[t]{2}{*}{ Variable } & \multicolumn{2}{|c|}{ No. (\%) } \\
\hline & ACP completed $(n=116)$ & Declined ACP $(n=166)$ \\
\hline Mean age (yr) & 82.6 & 80.2 \\
\hline Male gender & $34(29.3)$ & $41(24.7)$ \\
\hline \multicolumn{3}{|l|}{ Ethnicity } \\
\hline Chinese & $106(91.4)$ & $142(85.5)$ \\
\hline Malay & $5(4.3)$ & $12(7.2)$ \\
\hline Indian & $3(2.6)$ & $10(6.0)$ \\
\hline Other & $2(1.7)$ & $2(1.2)$ \\
\hline \multicolumn{3}{|l|}{ Current place of residence } \\
\hline Home & $113(97.4)$ & $159(95.8)$ \\
\hline Nursing home & $3(2.6)$ & $7(4.2)$ \\
\hline \multicolumn{3}{|l|}{ Function } \\
\hline Barthel Index for ADL $<80$ & $73(62.9)$ & $59(35.5)$ \\
\hline Very low ADL (score 0-24) & $33(28.4)$ & $16(9.6)$ \\
\hline Low ADL (score 25-49) & $16(13.8)$ & $11(6.6)$ \\
\hline Moderate reduced ADL (score 50-79) & $24(20.7)$ & $32(19.3)$ \\
\hline Independent ADL (score 80-100) & $43(37.1)$ & $107(64.5)$ \\
\hline Tube feeding & $6(5.2)$ & $1(0.6)$ \\
\hline \multicolumn{3}{|l|}{ Comorbidity } \\
\hline Hypertension & $92(79.3)$ & $133(80.1)$ \\
\hline Hyperlipidaemia & $72(62.1)$ & $105(63.3)$ \\
\hline Diabetes mellitus & $40(34.5)$ & $61(36.7)$ \\
\hline Ischaemic heart disease & $34(29.3)$ & $38(22.9)$ \\
\hline Cerebrovascular accident & $31(26.7)$ & $30(18.1)$ \\
\hline Chronic kidney disease & $26(22.4)$ & $38(22.9)$ \\
\hline Renal replacement therapy & $4(3.4)$ & $7(4.2)$ \\
\hline Underlying cognitive impairment & $66(56.9)$ & $68(41.0)$ \\
\hline Mild cognitive impairment & $0(0)$ & $5(3.0)$ \\
\hline Mild dementia & $15(12.9)$ & $23(13.9)$ \\
\hline Moderate dementia & $29(25.0)$ & $23(13.9)$ \\
\hline Moderately severe dementia & $10(8.6)$ & $12(7.2)$ \\
\hline Severe dementia & $12(10.3)$ & $5(3.0)$ \\
\hline Mean Charlson Cormobidity Index & 2.4 & 1.9 \\
\hline
\end{tabular}

ACP: Advance Care Planning; ADL: activities of daily living 
Table II. Univariate and multivariate logistic regression analyses of patient characteristics as potential predictors of agreement for ACP discussion.

\begin{tabular}{|c|c|c|c|c|}
\hline \multirow[t]{2}{*}{ Agreeable for ACP } & \multicolumn{2}{|c|}{ Univariate logistic regression analysis } & \multicolumn{2}{|c|}{ Multivariate logistic regression analysis } \\
\hline & OR $(95 \% \mathrm{Cl})$ & p-value & OR $(95 \% \mathrm{Cl})$ & p-value \\
\hline Age & $1.04(1.01-1.07)$ & $0.015^{*}$ & $1.03(0.99-1.06)$ & 0.101 \\
\hline Male gender & $1.26(0.74-2.15)$ & 0.389 & $1.26(0.71-2.23)$ & 0.433 \\
\hline Charlson Cormobidity Index & $1.15(1.02-1.31)$ & $0.024^{*}$ & $1.12(0.98-1.28)$ & 0.110 \\
\hline Barthel Index for ADL $<80$ & $3.05(1.86-4.99)$ & $<0.001^{*}$ & $2.22(1.27-3.87)$ & $0.005^{*}$ \\
\hline Diabetes mellitus & $0.91(0.55-1.49)$ & 0.696 & & \\
\hline Cerebrovascular accident & $1.72(0.97-3.06)$ & 0.063 & & \\
\hline Hypertension & $0.95(0.53-1.71)$ & 0.868 & & \\
\hline Hyperlipidaemia & $0.95(0.58-1.55)$ & 0.840 & & \\
\hline Ischaemic heart disease & $1.40(0.81-2.40)$ & 0.225 & & \\
\hline Chronic kidney disease & $1.01(0.57-1.78)$ & 0.980 & & \\
\hline Cognitive impairment & $2.08(1.28-3.38)$ & $0.003^{*}$ & $1.52(0.89-2.60)$ & 0.124 \\
\hline
\end{tabular}

* $p<0.05$ is statistically significant. ACP: advance care planning; ADL: activities of daily living; Cl: confidence interval; OR: odds ratio

Table III. Reasons for declining ACP discussion.

\begin{tabular}{|llll|}
\hline Reason & \multicolumn{3}{c|}{ No. (\%) } \\
\cline { 2 - 4 } & $\begin{array}{l}\text { Patients } \\
(\mathbf{n = 3 9 )}\end{array}$ & $\begin{array}{l}\text { FCG } \\
\text { (n= 23) }\end{array}$ & Total \\
\hline Doctor to decide & $5(8.1)$ & $2(3.2)$ & 7 \\
\hline Superstition & $2(3.2)$ & $1(1.6)$ & 3 \\
\hline Religious reason & $0(0)$ & $1(1.6)$ & 1 \\
\hline Inappropriate time & $2(3.2)$ & $4(6.5)$ & 6 \\
\hline No time to think/consider ACP & $2(3.2)$ & $3(4.8)$ & 5 \\
\hline $\begin{array}{l}\text { Lack of ACP awareness/ } \\
\text { understanding }\end{array}$ & $6(9.7)$ & $1(1.6)$ & 7 \\
\hline Not keen & $14(22.6)$ & $7(11.3)$ & 21 \\
\hline Deemed unnecessary & $4(6.5)$ & $2(3.2)$ & 6 \\
\hline Await other family members & $1(1.6)$ & $1(1.6)$ & 2 \\
\hline Too lazy to consider & $1(1.6)$ & $0(0)$ & 1 \\
\hline Patient would be horrified & $0(0)$ & $1(1.6)$ & 1 \\
\hline To think about it & $1(1.6)$ & $0(0)$ & 1 \\
\hline $\begin{array}{l}\text { Patient would be unable to recall } \\
\text { later on }\end{array}$ & $1(1.6)$ & $0(0)$ & 1 \\
\hline
\end{tabular}

ACP: advance care planning; FCG: family caregiver

The preferences of the remaining 116 patients or FCGs are shown in Table IV. A majority of patients and FCGs (79.3\%) opted for 'no CPR' and 82.8\% wanted limited medical intervention or comfort measures during medical deterioration. On univariate logistic regression analysis, older age (OR 1.18, 95\% Cl 1.08-1.28; $\mathrm{p}<0.001$ ), history of stroke (OR 4.83, 95\% $\mathrm{Cl} 1.06-22.00 ; \mathrm{p}=0.042$ ) and poorer functional status (OR 4.14, $95 \% \mathrm{Cl} 1.58-10.85 ; \mathrm{p}=0.004)$ were associated with declining CPR. The sample size was too small for multivariate logistic regression analysis.

With regard to preference for medical interventions, more than half of the patients were keen to have intravenous fluids $(74.1 \%)$, blood transfusion $(62.9 \%)$ and antibiotics $(73.3 \%)$ towards EOL, while half of them declined haemodialysis (50.9\%). In terms of tube feeding, nearly one-third of the cohort chose each option: oral feeding, tube feeding or undecided.
Table IV. Preference for cardiopulmonary resuscitation, medical intervention or life-sustaining treatments for EOL care $(n=116)$.

\begin{tabular}{|ll|}
\hline Preference & No. (\%) \\
\hline Cardiopulmonary resuscitation & \\
\hline Proceed & $23(19.8)$ \\
\hline Do not proceed & $92(79.3)$ \\
\hline Undecided & $1(0.9)$ \\
\hline Medical intervention & $16(13.8)$ \\
\hline Full & $96(82.8)$ \\
\hline Limited or comfort & $4(3.4)$ \\
\hline Undecided & \\
\hline Mode of feeding & $37(31.9)$ \\
\hline Oral & $40(34.5)$ \\
\hline Tube & $39(33.6)$ \\
\hline Undecided & \\
\hline Antibiotics & $85(73.3)$ \\
\hline Yes & $4(3.4)$ \\
\hline No & $27(23.3)$ \\
\hline Undecided \\
\hline Haemodialysis \\
\hline Yes & $21(18.1)$ \\
\hline No & $59(50.9)$ \\
\hline Undecided & $36(31.0)$ \\
\hline Blood transfusion & $29(25.0)$ \\
\hline Yes & $25(21.6)$ \\
\hline No & \\
\hline Undecided & \\
\hline Intravenous fluid & \\
\hline Yes & \\
\hline No & \\
\hline Undecided & \\
\hline & \\
\hline
\end{tabular}

EOL: end of life

In terms of their preference for place of medical care and death (Table V), most (48.3\%) patients preferred to be transferred to an acute hospital in the event of deterioration. The same proportion of elderly people were undecided about their preferred 


Table V. Preferred places of medical treatment and death in the
event of deterioration ( $\mathbf{n = 1 1 6 ) .}$
\begin{tabular}{|ll|}
\hline Preference & No. (\%) \\
\hline Place of treatment & $12(10.3)$ \\
\hline $\begin{array}{l}\text { Remain in my own home/nursing home/hospice/ } \\
\text { hospital }\end{array}$ & $4(3.4)$ \\
\hline $\begin{array}{l}\text { Trial of treatment at current location (home/nursing } \\
\text { home/hospice) before considering transfer to } \\
\text { hospital }\end{array}$ & $56(48.3)$ \\
\hline \begin{tabular}{l} 
Transfer to acute hospital \\
\hline Undecided
\end{tabular} & $44(37.9)$ \\
\hline Place of death & $21(18.1)$ \\
\hline Hospital & $35(30.2)$ \\
\hline Home & $2(1.7)$ \\
\hline Hospice & $2(1.7)$ \\
\hline Nursing home & $56(48.3)$ \\
\hline Undecided &
\end{tabular}

place of demise (48.3\%). One-third of the respondents preferred to pass on at home $(30.2 \%)$, while fewer preferred to pass on in an acute hospital (18.1\%).

\section{DISCUSSION}

This study explored the novel addition of ACP as part of CGA for frail hospitalised geriatric patients and demonstrated the utility of doing this together with its resulting outcomes. CGA requires establishing good communication with and understanding of patients and/or their FCGs; the addition of ACP in the CGA process leverages on this relationship. A local retrospective review study on EOL care in an acute tertiary hospital demonstrated inadequate commitment by doctors towards do-not-resuscitate orders and limiting life-sustaining therapies and interventions, together with insufficient EOL discussions and suboptimal palliative care. ${ }^{(12}$ Integrating ACP into CGA is not only sensible and logical, but also makes ACP part of the daily ward rounds for geriatric medicine teams and enables team doctors to think about and actively partake in ACP discussions during their patient and/or FCG encounters. In addition, it is important to note that a large proportion of the study cohort $(56.9 \%$ of ACP completed group, $41.0 \%$ of ACP declined group) had some form of underlying cognitive impairment, ranging from mild cognitive impairment to dementia. A 2012 joint recommendation by the Royal College of Physicians and Royal College of Nursing on principles for best practice in ward rounds noted that "inpatient populations increasingly consist of frail older patients, with estimates of prevalence of dementia as high as 25\%" and recommended that such patients be supported to make decisions about their care as far as possible. ${ }^{(13)}$ In this aspect, conducting ACP as part of CGA for demented frail elderly people can help these patients and their FCGs make supported EOL care decisions in the best interests of the patients.

A majority of our patients had no prior ACP awareness. In terms of receptiveness, more than half of our patients declined ACP discussion. Of the decliners, $37.3 \%$ provided reasons for rejection. For patients and FCGs, 'not keen' was the most common reason not to engage in ACP discussion, followed by 'deferring to doctor's decision' and 'lack of ACP awareness/ understanding'. Taken as a whole, this could stem from poor awareness and understanding of the role and importance of ACP. Other contributing factors included the setting and timing of ACP discussion. While acute hospitalisation may not be the best setting and time to conduct $\mathrm{ACP}$, the fact remains that for most patients and FCGs in Singapore, the acute hospital is the first site of encounter for ACP discussion for frail geriatric patients. Hospital is also often the place where FCGs realise the gravity and severity of the patient's medical condition. Overall, despite the limitations, ACP discussions during hospitalisation maintain their utility in initiating the conversation and planting the concept of ACP in the minds of patients and FCGs.

The large proportion of decliners constitutes an important group that has often been neglected in ACP implementation. Currently, patients or FCGs who initially decline ACP discussion are not followed up on. A prospective study showed that elderly people reported less desire to receive life-sustaining treatment soon after hospitalisation, but this desire returned to near prehospitalisation levels months after discharge. ${ }^{(14)}$ This finding suggested that care choices by elderly may be susceptible to contextual changes in health status. Hence, in our local context, follow-up contact for decliners in the form of home visits by dedicated ACP teams post hospitalisation (when time, ambience and privacy are assured) would enable the discussion to continue in a more in-depth and unhurried manner. Devoting more resources into developing these teams to follow up with decliners post hospitalisation would thus be a consideration.

Poorer functional status, as defined by a modified Barthel Index $<80$, was found to be significantly associated with agreeing to ACP discussion. This likely reflects the increased burden and decreased quality of life encountered by patients or FCGs who were thus more receptive to ACP discussion. A community survey of elderly people in the United States demonstrated that those with poorer functional status were more likely to have engaged in ACP, whereas a study in Singapore demonstrated that most adults aged over 50 years chose quality of life over length of life when compared with advanced cancer patients. ${ }^{(15,16)}$ Such inclination increased with age. While relying solely on a modified Barthel Index $<80$ to stratify patients would be an oversimplification, our study results show that stratification by functional status could indeed be considered and utilised as part of a collective strategy to help more patients achieve successful completion of ACP discussion. Certainly, further research that explores the importance and role of functional status in determining successful ACP discussions in the local setting is warranted.

Opinions were divided over EOL care choices. For CPR, a majority was clear that they did not want $\mathrm{CPR}$ at EOL, with only $1(0.9 \%)$ patient being undecided. For the remaining EOL care choices (medical interventions, mode of feeding, antibiotics, haemodialysis, blood transfusion and intravenous fluid), the proportions of 'undecided' responses were greater, ranging 
from $3.4 \%$ to $33.6 \%$. A cross-sectional questionnaire survey on Chinese inpatients aged 60 years and above in Hong Kong yielded comparable findings in terms of resuscitation $-80 \%$ of patients declined CPR and $81 \%$ declined artificial ventilation, while smaller proportions (range $40 \%-57 \%$ ) opted for other EOL care choices, with $10 \%$ being undecided. ${ }^{(17)}$ Overall, this likely reflects the relatively greater awareness of CPR among laypeople, as compared to other EOL care choices, and highlights the need for greater public education on the matter. The importance of such education programmes cannot be overemphasised. EOL care choices that are not of immediate concern to elderly people will likely result in pseudo-participation, in which they could make EOL care choices without really knowing what is at stake.

In our study, there were similar findings for both 'preferred place of medical treatment and care in event of deterioration' and 'preferred place of death'. Notably, a majority (97.4\%) of $\mathrm{ACP}$ respondents were residing in their homes in the community. A large proportion (48.3\%) chose to transfer to an acute hospital in the event of deterioration towards EOL, while a slightly smaller proportion (37.9\%) was undecided. At the same time, a majority $(48.3 \%)$ of respondents were undecided as to where they would prefer to pass away. These findings highlight a few important points. One, patients still preferred to go to acute hospitals towards EOL, trusting in their standard of care. Two, choosing to go to an acute hospital during deterioration may take precedence over their choice of place of death. Patients appeared keener to receive some form of escalated care in the acute setting without realising that similar care can be achieved in the nursing homes or at home. At present, such a mindset appears entrenched for most patients and FCGs, and this trend can only be reversed with time, continuous education and the building up of more teams for home-based EOL care.

The study had some limitations. Our sample size was relatively small and among those who declined, more than half did not provide reasons for refusing ACP. For those who agreed to ACP discussion, 29 people did not complete the discussion. Hence, the EOL care choices and reasons for refusal in our cohort were not fully captured. In terms of efficacy of integrating ACP into CGA, although $37.3 \%$ of the study completed ACP, we had no previous data for comparison. This limitation could be overcome by a study with a larger sample size and a control group, which should be considered for future research. Furthermore, the stability of EOL care preferences over time was not evaluated. A systemic review of stability of EOL preference showed that preference of stability over time was generally associated with inpatients and unwell outpatients rather than stable elderly people. ${ }^{(18)}$ Future studies evaluating the stability of EOL care preferences, both during hospitalisation and post hospitalisation, in relation to changes in health status can be considered.
In conclusion, ACP is an evolving challenge in a rapidly greying inpatient population. Our study has demonstrated the feasibility and utility of integrating ACP as part of CGA for frail geriatric patients. It has explored the reasons for declining ACP discussion in the inpatient setting and highlighted the types of EOL care choices, including uncertainty and indecision among patients and FCGs. Stratification of patients by functional status, greater public education on EOL care choices (besides CPR) and dedicated ACP teams who conduct follow-up visits on decliners post hospitalisation will likely yield more positive outcomes and add new dimensions to a systematic approach towards ACP advocacy among hospitalised frail elderly people.

\section{REFERENCES}

1. Rao JK, Anderson LA, Lin FC, Laux JP. Completion of advance directives among U.S. consumers. Am J Prev Med 2014; 46:65-70.

2. Detering KM, Hancock AD, Reade MC, Silvester W. The impact of advance care planning on end of life care in elderly patients: randomised controlled trial. BMJ 2010; 340:c1345.

3. Lien Foundation. Death Attitudes Survey 8 April 2014. http://lienfoundation. org/sites/default/files/Gen\%20Pop\%20Findings\%20Report\%20-\%20Full\%20 REPORT\%20\%28Website\%29_0.pdf. Accessed August 12, 2019.

4. Ng R, Chan S, Ng TW, Chiam AL, Lim S. An exploratory study of the knowledge, attitudes and perceptions of advance care planning in family caregivers of patients with advanced illness in Singapore. BMJ Support Palliat Care 2013; 3:343-8.

5. Low JA, Ng WC, Yap KB, Chan KM. End-of-life issues--preferences and choices of a group of elderly Chinese subjects attending a day care centre in Singapore. Ann Acad Med Singapore 2000; 29:50-6.

6. Ang GC, Zhang D, Lim KH. Differences in attitudes to end-of-life care among patients, relatives and healthcare professionals. Singapore Med J 2016; 57:22-8.

7. Ng CW, Cheong SK, Govinda Raj A, Teo W, Leong I. End-of-life care preferences of nursing home residents: results of a cross-sectional study. Palliat Med 2016; 30:843-53.

8. Rubenstein LZ, Stuck AE, Siu AL, Wieland D. Impact of geriatric evaluation and management programs on defined outcomes: overview of the evidence. J Am Geriatr Soc 1991; 39(9 Pt 2):8S-16S; discussion 17S-18S

9. Kim YS, Escobar GJ, Halpern SD, et al. The natural history of changes in preferences for life-sustaining treatments and implications for inpatient mortality in younger and older hospitalized adults. J Am Geriatr Soc 2016; 64:981-9.

10. Matzen LE, Jepsen DB, Ryg J, Masud T. Functional level at admission is a predictor of survival in older patients admitted to an acute geriatric unit. BMC Geriatr 2012; 12:32.

11. Reisberg B. Functional assessment staging (FAST). Psychopharmacol Bull 1988; 24:653-9.

12. Phua J, Kee AC, Tan A, et al. End-of-life care in the general wards of a Singaporean hospital: an Asian perspective. J Palliat Med 2011; 14:1296-301.

13. Royal College of Physicians, Royal College of Nursing. Ward rounds in medicine: principles for best practice. October 2012. Available at: https:// www.rcplondon.ac.uk/projects/outputs/ward-rounds-medicine-principles-bestpractice. Accessed January 17, 2018.

14. Ditto PH, Jacobson JA, Smucker WD, Danks JH, Fagerlin A. Context changes choices: a prospective study of the effects of hospitalization on life-sustaining treatment preferences. Med Decis Making 2006; 26:313-22.

15. Bischoff KE, Sudore R, Miao Y, Boscardin WJ, Smith AK. Advance care planning and the quality of end-of-life care in older adults. J Am Geriatr Soc 2013; 61:209-14.

16. Malhotra C, Xiang L, Ozdemir S, et al. A comparison of attitudes toward length and quality of life between community-dwelling older adults and patients with advanced cancer. Psychooncology 2017; 26:1611-7.

17. Ting FH, Mok E. Advance directives and life-sustaining treatment: attitudes of Hong Kong Chinese elders with chronic disease. Hong Kong Med J 2011; 17:105-11.

18. Auriemma CL, Nguyen CA, Bronheim R, et al. Stability of end-of-life preferences: a systemic review of the evidence. JAMA Intern Med 2014; 174:1085-92. 